\title{
Respiratory gating in PET/CT: A step in the right direction
}

\author{
Tinsu Pan, PhD \\ ${ }^{\text {a }}$ Houston, TX
}

Received Aug 3, 2016; accepted Aug 9, 2016

doi:10.1007/s12350-016-0647-4

\section{See related article, pp. 407-415}

Respiratory motion is inevitable in cardiac imaging with PET as the rest or stress myocardial perfusion imaging (MPI) takes about 6 minutes with ${ }^{82} \mathrm{Rb}$ and 10 to 15 minutes with ${ }^{13} \mathrm{~N}$-ammonia and viability imaging 10 to 30 min with ${ }^{18}$ F-FDG. ${ }^{1}$ Figure 1 illustrates an example of respiratory motion between the end-inspiration and end-expiration phases in an ${ }^{18} \mathrm{~F}$-FDG scan. All newer PET scanners are with CT and without transmission line sources. ${ }^{2}$ CT has shortened the time for the transmission scan for attenuation correction of the PET data from minutes to seconds and could also provide the important information of calcium scores and contrast-enhanced coronary artery CT images to help diagnose heart disease. CT, however, introduces a new problem of potential mis-registration of the CT and PET data due to its fast scan speed resulting in each CT image being a snapshot or a single phase of the heart in respiratory motion. A series of continuous respiratory phases of snapshot CT images may not be suitable for attenuation correction of the PET data. It is important to mitigate the impact of respiratory motion to improve cardiac PET image quality.

In the article by Ichikawa et al, the authors proposed an abdominal restriction technique by applying an abdominal belt to restrict respiratory motion in MPIPET. They wrapped a $20-\mathrm{cm}$ abdominal belt around the abdomen of 8 male healthy volunteers (BMI 23 \pm 2 and ages $55 \pm 17$ years) at end-expiration to restrict their respiratory motion. They reported that the motion of the heart in the cranio-caudal direction was significantly reduced from $12.1 \pm 6.1 \mathrm{~mm}$ without the belt to

Reprint requests: Tinsu Pan, Houston, TX; TPan@mdanderson.org J Nucl Cardiol 2018;25:416-8.

$1071-3581 / \$ 34.00$

Copyright (C) 2016 American Society of Nuclear Cardiology.
$8.1 \pm 7.1 \mathrm{~mm}$ with the belt and that this restriction was well tolerated in the 8 healthy volunteers and subsequent 53 patients (ages $70 \pm 10$ ). The authors also reported a small but statistically significant improvement of a qualitative visualization score of $0.42(0.29$ to 0.71$)$ and $0.71(0.33$ to 1.04$)$ in the anterior and inferior walls, respectively, and no change in the lateral walls in a scale of $0=$ normal to $4=$ defect. Reduction of respiratory motion did not have an impact on the assessment of cardiac functions of ejection fraction or left ventricular end-diastolic or end-systolic volumes. In conclusion, the abdominal belt technique was suggested to be an effective way of reducing the impact of respiratory motion in MPI-PET or even MPI-SPECT imaging.

Some limitations were noted. The data were only from rest MPI-PET, not stress MPI-PET; there was no quantitative blood flow measurement; there was no standard for adjusting the abdominal belt other than 'the belt was tightened as much as possible without causing discomfort to the patient.' It was not clear if this 'tolerable' procedure can be translated into a 'comfortable' procedure, which may be the best indicator for success of an MPI-PET scan, and which can be best represented by the regular or normal breathing patterns recorded, but not reported in the study. The belt may need to be loosened between the rest and stress MPI studies to improve patient comfort when the total duration of the rest and stress studies can last over 40 minutes for ${ }^{13} \mathrm{~N}$ ammonia and many patients cannot keep still after 20 minutes on the imaging couch.

In addition to the proposed abdominal restriction technique, there are other techniques from radiation therapy that are used to mitigate the respiratory motion in treating mobile tumors in the thorax or abdomen. Abdominal compression, similar to the proposed abdominal belt to restrict respiratory motion, can restrict the abdominal motion by applying an abdominal plate against the abdomen under a controlled pressure. ${ }^{3}$ However, the device is bulky, needs to be 
setup on a flat-couch, and may not be suitable in an MPI-PET session with EKG leads and wires. Another way of reducing the respiratory motion is to have the patient wear goggles to watch a real-time video of the patient's respiration level and to ask the patient to exercise respiration in a predefined range. Some may also play a recording of breathing instructions to coach the patient to breath in a shallow manner and in a certain frequency to make respiratory motion regular. Abdominal compression, wearing goggles, and playing breathing recordings have been practiced in radiation therapy. However, it may be impractical to expect all patients to follow any or a combination of these somewhat intrusive procedures. Some may tolerate one procedure better than another, and some may not be able to tolerate any procedure without increasing anxiety to cause unintended increase in motion. A patient could also do perfectly during a training session and do very poorly during the imaging session or vise versa. The best results may come from the patients without any breathing instructions or restrictive devices because most patients breathe shallowly in their natural states.

Another important degrading factor of respiratory motion in MPI-PET is the potential mis-registration of the CT and PET images due to mismatch of their temporal resolution. The transmission map and emission data of a stand-alone PET do not have mis-registration artifacts at the diaphragm level. ${ }^{4}$ Current PET/CT only matches the spatial resolution by blurring CT to match PET. The CT image is typically reconstructed with less than 1 second of data, whereas PET image is from many respiratory cycles over several minutes. Average CT, derived from either a cine CT or low pitch helical CT scan of high-speed gantry rotation over a respiratory cycle, has a similar temporal resolution as PET and can mimic the transmission map from the transmission line sources of a stand-alone PET. ${ }^{5-7}$
Average CT can improve registration of CT with PET and has been implemented on several vendors of PET/CT scanners. Unfortunately, the average CT from vendors requires respiratory gating, typically used in four-dimensional CT $(4 \mathrm{D}-\mathrm{CT})^{8}$ for radiation therapy treatment planning of the lung tumor, complicating the clinical solution, and making it impractical because most PET/CT scanners are either without respirator gating hardware or respiratory gating was deemed too complex to be applied in a clinical setting. It will be beneficial to make the average CT available in cardiac PET without respiratory gating as it has been demonstrated in the original proposal of average CT. ${ }^{9}$ The patient breathes the same way for average CT and (average) PET. The radiation dose of average $\mathrm{CT}$ can be less than $1 \mathrm{mSv}$. Average CT takes care of the nonrigid breathing motion in CT, and rigid-body-translation software takes care of patient motion between CT and PET during the MPIPET scan.

If neither the abdominal belt nor average CT can be utilized, a practical and popular approach is to CT scan at or near end-expiration to match the CT and PET images. We typically spend more time in expiration than in inspiration. The PET data will be weighted more toward the end-expiration and will have a better chance to match with CT at or near end-expiration, which can be implemented with proper patient coaching. The scan time with a 16-slice CT of 2-cm X-ray collimation can be as short as 3 seconds for a coverage of $15 \mathrm{~cm}$ or one bed of PET scan field of view, which makes it relatively easy for the patient to hold breath at or near end-expiration in a fast CT scan of just a few seconds. Coordination between the patient and the technologist is important for the success of breath-hold at or near endexpiration.

Most vendors provide a rigid-body-translation software for registration of the CT and PET images to ensure that PET activities do not overlap with the low-

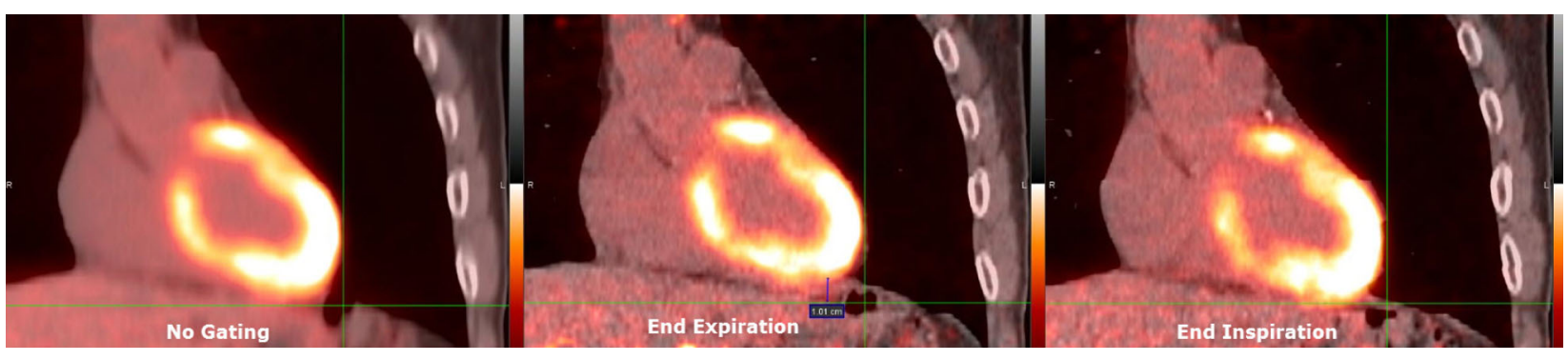

Figure 1. An ${ }^{18}$ F-FDG cardiac scan with respiratory gating of both PET and CT. The fusion of PET and average CT (left), the fusion of end-expiratory PET and end-expiratory CT (center), and the fusion of end-inspiratory PET and end-inspiratory CT (right). There was about 1-cm diaphragm motion between the end-expiratory and end-inspiratory phases. 
density lung region to avoid under-correction of the PET activities due to photon attenuation. Although respiration is a nonrigid motion, most mis-registration problems can be fixed with rigid body translation. However, rigid body translation may not be able to ensure the PET activities stay within the heart region depicted in CT due to the nonrigid nature of the respiratory motion.

Looking into the future, the most ideal solution to manage respiratory motion is to acquire data in listmode, use a data-driven self-gating technique to gate the PET activities into multiple bins over a respiratory cycle, ${ }^{10}$ and use deformable registration of the multiple bins of PET activities into a single bin to preserve the count statistics in PET imaging. All of these happen without any hardware gating and under the assumption that the patient breathes regularly during the MPI-PET. Average CT over one breath cycle or a single helical CT at or near end-expiration can also be registered to the multiple bins of PET for attenuation correction. Of course, any effort to improve the MPI-PET will need to be clinically proven to benefit the patients at a relatively low cost in implementation and usage.

\section{References}

1. Dilsizian V, et al. ASNC imaging guidelines/SNMMI procedure standard for positron emission tomography (PET) nuclear cardiology procedures. J Nucl Cardiol. 2016. doi:10.1007/s12350016-0522-3.

2. Mawlawi O, Townsend DW. Multimodality imaging: an update on PET/CT technology. Eur J Nucl Med Mol Imaging. 2009;36(Suppl 1):S15-29.

3. Huang TC, et al. Respiratory motion reduction in PET/CT using abdominal compression for lung cancer patients. PLoS One. 2014;9(5):e98033.

4. Osman MM, et al. Respiratory motion artifacts on PET emission images obtained using CT attenuation correction on PET-CT. Eur J Nucl Med Mol Imaging. 2003;30(4):603-6.

5. Pan T, et al. Attenuation correction of PET cardiac data with lowdose average CT in PET/CT. Med Phys. 2006;33(10):3931-8.

6. Alessio AM, et al. Cine CT for attenuation correction in cardiac PET/CT. J Nucl Med. 2007;48(5):794-801.

7. Cook RA, et al. Respiration-averaged CT for attenuation correction in canine cardiac PET/CT. J Nucl Med. 2007;48(5):811-8.

8. Pan T, et al. 4D-CT imaging of a volume influenced by respiratory motion on multi-slice CT. Med Phys. 2004;31(2):333-40.

9. Gould KL, et al. Frequent diagnostic errors in cardiac PET/CT due to misregistration of CT attenuation and emission PET images: a definitive analysis of causes, consequences, and corrections. J Nucl Med. 2007;48(7):1112-21.

10. Kesner AL. Current state of data-based gating technology in PET imaging. Eur J Nucl Med Mol Imaging. 2011;38(4):785. 